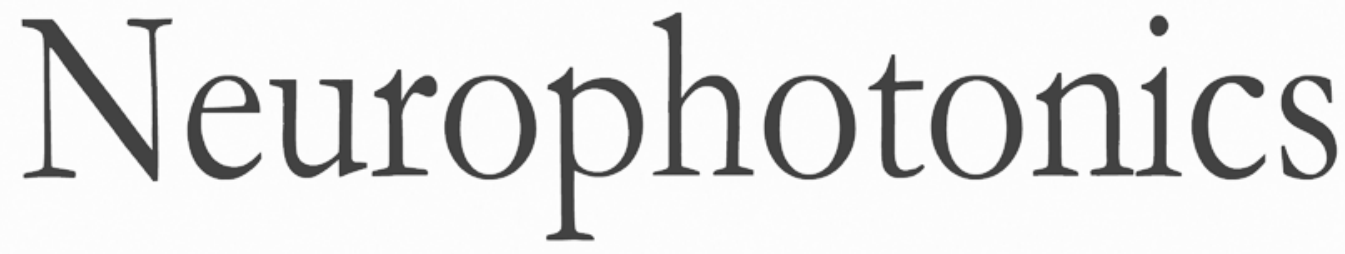

\title{
Noninvasive detection of acute cerebral hypoxia and subsequent matrix-metalloproteinase activity in a mouse model of cerebral ischemia using multispectral-optoacoustic- tomography
}

\author{
Ruiqing Ni \\ Markus Vaas \\ Wuwei Ren \\ Jan Klohs
}




\title{
Noninvasive detection of acute cerebral hypoxia and subsequent matrix-metalloproteinase activity in a mouse model of cerebral ischemia using multispectral-optoacoustic-tomography
}

\author{
Ruiqing Ni, Markus Vaas, Wuwei Ren, and Jan Klohs* \\ University of Zurich and ETH Zurich, Institute for Biomedical Engineering, Zurich, Switzerland
}

\begin{abstract}
Oxygen metabolism and matrix metalloproteinases (MMPs) play important roles in the pathophysiology of cerebral ischemia. Using multispectral optoacoustic tomography (MSOT) imaging, we visualized in vivo changes in cerebral tissue oxygenation during $1 \mathrm{~h}$ of transient middle cerebral artery occlusion (tMCAO) and at $48 \mathrm{~h}$ after reperfusion together with MMP activity using an MMP-activatable probe. The deoxyhemoglobin, oxyhemoglobin, and MMP signals were coregistered with structural magnetic resonance imaging data. The ipsi-/contralateral ratio of tissue oxygen saturation $\left(\mathrm{SO}_{2}\right)$ was significantly reduced during $1 \mathrm{~h}$ of tMCAO and recovered after $48 \mathrm{~h}$ of reperfusion in tMCAO compared with sham-operated mice ( $n=8$ to 10 per group). A higher ipsi-/contralateral MMP signal ratio was detected at $48 \mathrm{~h}$ after reperfusion in the lesioned brain regions of tMCAO compared with the sham-operated animal ( $n=4$ to 6 per group). Ex vivo near-infrared fluorescence imaging of MMP signal in brain slices was used to validate in vivo MSOT measurements. In conclusion, noninvasive MSOT imaging can provide visualization of hemodynamic alterations and MMP activity in a mouse model of cerebral ischemia. ๑ 2018 Society of Photo-Optical Instrumentation Engineers (SPIE) [DOI: 10.1117/1.NPh.5.1.015005]
\end{abstract}

Keywords: multispectral optoacoustic imaging; cerebral ischemia; matrix metalloproteinases; magnetic resonance imaging; hemodynamics; animal model.

Paper 17122RR received Oct. 16, 2017; accepted for publication Jan. 12, 2018; published online Feb. 28, 2018.

\section{Introduction}

Hemodynamic alterations and the subsequent inflammatory responses are important pathophysiological processes following cerebral ischemia. Acute reduction of cerebral blood flow (CBF) results in a shortage of glucose and oxygen to the supplied area. In the core of the ischemic territory, with the most severe reduction in $\mathrm{CBF}$, irreversible tissue damage occurs within minutes to hours after the occlusion due to depletion of adenosine triphosphate and energy failure. ${ }^{1}$ Surrounding the ischemic core, referred to as ischemic penumbra, the brain tissue is still partially perfused although at a reduced rate. Neurons in the penumbra also face major challenges to their survival, such as excitotoxicity, peri-infarct polarizations, inflammation and apoptosis, leading to a secondary tissue damage and expansion of the lesion. ${ }^{2}$ As therapeutic interventions for ischemic stroke mainly aim to restore tissue oxygenation and $\mathrm{CBF}$, measurement of cerebral tissue oxygenation thus provides crucial information.

Matrix metalloproteinases (MMPs), in particular MMP-2 and MMP-9, are expressed and released during the inflammatory responses in different cell types in the brain, ${ }^{3}$ including endothelial cells, microglia, astrocytes, neurons, and neutrophils. ${ }^{4}$ MMP activity exerts differential effects in the central nervous system and has been implicated to partake in blood-brain barrier impairment, edema formation, hemorrhagic transformation, and neuronal cell death. In regards to time scale, MMP activity

*Address all correspondence to: Jan Klohs, E-mail: klohs@ biomed.ee.ethz.ch contributes to the expansion of the core lesion during the acute phase but is also involved in tissue regeneration and angiogenesis during the chronic stage after cerebral ischemia. ${ }^{5-11}$ In vivo visualization of MMP activity along with measuring changes in tissue oxygenation is therefore valuable for understanding the pathophysiological dynamics of cerebral ischemia and for monitoring the treatment response. ${ }^{12}$

Optoacoustic imaging operates on the local thermoelastic expansion of photoabsorbing molecules, following the absorption of transient incident light. The thermoelastic expansion generates broadband ultrasonic waves, which can be sensed by ultrasound transducers. Advanced imaging schemes require the placement of multiple detectors around the tissue for tomographic reconstruction of the location of the photoabsorbing material. ${ }^{13}$ Using multiple wavelengths for illuminating and spectral unmixing algorithms, multispectral optoacoustic tomography (MSOT) resolves multiple channels of photoabsorbing molecules in tissue. ${ }^{14}$ Thus, MSOT has become a promising imaging modality for biomedical research and has found applications in neuroimaging models of brain diseases. In biological tissue, the major absorbers are melanin, water, and hemoglobin, which can serve as endogenous contrast agents for MSOT. Monitoring concentration of oxygenated and deoxygenated blood using MSOT has provided information on cerebral tissue oxygenation in the rat and mouse brain at rest and during functional activation ${ }^{15-19}$ and has revealed an oxygenation deficit 
in the mouse brain after the transient middle cerebral artery occlusion (tMCAO) in the model of cerebral ischemia. ${ }^{20}$

A number of contrast agents for MSOT, which enable cellular and molecular imaging and are based on fluorescent dyes and proteins and plasmonic nanoparticles, have been developed..$^{21,22}$ The feasibility to detect MMPs activity with MSOT has been previously demonstrated in human carotid plaques ex vivo with MMP-activatable probes ${ }^{22,23}$ and targeted gold nanorods. ${ }^{24}$

In the current study, we demonstrated the detection of tissue oxygenation alterations and MMP activity in the mouse brain after focal cerebral ischemia and reperfusion with MSOT in vivo. To overcome the limited soft tissue contrast of MSOT in the brain, we implemented a framework for coregistering MSOT data with diffusion weighted imaging (DWI) and $\mathrm{T}_{2}$-weighted magnetic resonance imaging (MRI) data, structurally demarcating also the ischemic lesion in the hyperacute and acute phase. Ex vivo validation of MMP activity was made with near-infrared fluorescence (NIRF) imaging.

\section{Methods}

\subsection{In Vitro Activation of the MMP-Activatable Probe}

The MMP-activatable probe (MMPSense ${ }^{\mathrm{TM}}$ 680, Perkin Elmer) is predominantly activated by MMP-9, -2 and -13 , but to a lesser extent also by other MMPs. ${ }^{25}$ The MMP-activatable probe $(0.2 \mu \mathrm{mol} / \mathrm{L})$ was incubated with $4.5-\mu \mathrm{g}$ human recombinant MMP-9 (Oncogene Research Products, California, USA) at $37^{\circ} \mathrm{C}$ in a buffer containing $50 \mathrm{mmol} / \mathrm{L}$ Tris $\mathrm{HCl} \mathrm{pH} \mathrm{7.5,}$ $10 \mathrm{mmol} / \mathrm{L} \mathrm{CaCl}_{2}, 100 \mathrm{mmol} / \mathrm{L} \mathrm{NaCl}$, and $0.005 \%$ Brij-35 (Sigma-Aldrich). Tubes ( $3 \mathrm{~mm}$ diameter, 4 to $5 \mathrm{~cm}$ lengths) were filled with either $200 \mu \mathrm{l}$ activated probe or nonactivated probe for NIRF imaging. For obtaining MSOT absorbance spectra and standard curve of activated MMP, $1 \mu \mathrm{M}$ MMP-activatable probe and $100 \mu \mathrm{M}$ of trypsin solution from porcine pancreas (Sigma-Aldrich, Switzerland) in a 1:2 dilution series were used. The solutions were filled in tubes of the same type used ( $3 \mathrm{~mm}$ diameter, 4 to $5 \mathrm{~cm}$ lengths) in NIRF imaging with the Maestro 500 multispectral imaging system (Cambridge Research \& Instruments Inc.) and placed in 2-cm-diameter cylindrical phantoms made of $2 \%$ agarose (solidifying at $20^{\circ} \mathrm{C}$; Sigma-Aldrich) mixed with $5 \%$ intralipid.

\subsection{Animal Model}

All procedures conformed to the national guidelines of the Swiss Federal Act on Animal Protection and were approved by the Cantonal Veterinary Office Zurich (Permit Number: 18-2014). Thirty-nine male C57BL/6J mice (Janvier, France), weighing 20 to $25 \mathrm{~g}, 8$ to 10 weeks of age, were used including 19 sham-operated mice ( 2 died during surgery) and 20 tMCAO mice. Data for one tMCAO was excluded due to strong pigment on the mouse head interfering with MSOT and NIRF data acquisition. To assess cerebral oxygenation during the hyperacute phase (i.e., vessel occlusion in tMCAO group), tMCAO $(n=9)$ and sham-operated mice $(n=9)$ underwent in vivo MSOT and DW MRI and triphenyltetrazolium chloride (TTC) for validation after in vivo imaging. Considering that MSOT measurement and depilating gel on the skin might influence the inflammation status of the mice, another batch of tMCAO $(n=10)$ and sham-operated mice $(n=8)$ was used to assess for cerebral oxygenation in the acute stage at $48 \mathrm{~h}$ after reperfusion: in vivo MSOT, $\mathrm{T}_{2}$-weighted MRI, ex vivo NIRF, and histology staining sequentially. Additionally, six tMCAO mice and four sham-operated mice were assessed for tissue oxygenation at $48 \mathrm{~h}$ after surgery and were also injected intravenously with $300 \mu \mathrm{l} 4 \mathrm{nM}$ of MMPSense ${ }^{\mathrm{TM}} 680$ after $1 \mathrm{~h}$ tMCAO. Animals were assessed with MSOT for detecting MMP activity after reperfusion or sham-operation. Three tMCAO mice without MMP probe injection were assessed with MSOT and served as controls.

\subsection{Transient Middle Cerebral Artery Occlusion}

Surgeries for tMCAO and sham were performed as described before. $^{26}$ Anaesthesia was initiated using 3\% isoflurane (Abbott, Cham, Switzerland) in a 1:4 oxygen/air mixture and maintained at $2 \%$. Before the surgical procedure, a local analgesic (Lidocaine, $0.5 \%, 7 \mathrm{mg} / \mathrm{kg}$, Sintectica S.A., Switzerland) was administered subcutaneously (s.c.). Temperature was kept constant at $36.5^{\circ} \mathrm{C} \pm 0.5^{\circ} \mathrm{C}$ with a feedback controlled warming pad system. All surgical procedures were performed in less than 15 min. After surgery, buprenorphine was administered as s.c. injection (Temgesic, $0.1 \mathrm{mg} / \mathrm{kg}$ b.w.) and then twice s.c. every 6 to $8 \mathrm{~h}$ on the day of surgery and was supplied thereafter via the drinking water $(1 \mathrm{mg} / \mathrm{kg})$ until $48 \mathrm{~h}$.

\subsection{Multispectral Optoacoustic Tomography}

A MSOT inVision 128 imaging system (iThera Medical, Germany) was used as described previously. ${ }^{26}$ Briefly, a tunable (680 to $980 \mathrm{~nm}$ ) optical parametric oscillator pumped by an Nd:YAG laser provides 9-ns excitation pulses at a framerate of $10 \mathrm{~Hz}$ with a wavelength tuning speed of $10 \mathrm{~ms}$ and a peak pulse energy of $100 \mathrm{~mJ}$ at $730 \mathrm{~nm}$. Ten arms, each containing an optical fiber bundle, provide even illumination of a ringshaped light strip with a width of $\sim 8 \mathrm{~mm}$. For ultrasound detection, 128 cylindrically focused ultrasound transducers with a center frequency of $5 \mathrm{MHz}(60 \%$ bandwidth), organized in a concave array of 270-deg angular coverage and a curvature radius of $4 \mathrm{~cm}$, were used.

For a phantom MSOT measurement, the phantom was fixed into the supplied rigid phantom holder and placed into the imaging chamber of the MSOT system, filled with $36.5^{\circ} \mathrm{C}$ warm water. Phantom measurements using different concentrations of trypsin and MMP activatable probe were used to generate MMP MSOT absorbance spectra. After equilibrating of the phantom temperature for $5 \mathrm{~min}$, imaging was performed at 45 wavelengths from 680 to $900 \mathrm{~nm}$ with 5-nm increment. Principal component analysis based on Ref. 27 was used for calculating the absorbance spectra of MMP and used in the unmixing and multispectral analysis of the phantom and tMCAO-/shamoperated mice. For the phantom activated and unactivated human recombinant MMP-9 with MMP activatable probe, imaging was performed at 10 wavelengths (i.e., 680, 685, 690, $695,700,715,730,760,800$, and $850 \mathrm{~nm}$ ) at 35 to 40 consecutive slices with a step size of $0.1 \mathrm{~mm}$ and 10 averages. For in vivo MSOT measurement, animals were anesthetized with $4 \%$ and maintained at $1.5 \%$ isoflurane in a $1: 4$ oxygen/air mixture supplied via a nose cone. Mice were depilated around the head and placed in a mouse holder in the supine position. Preheated ultrasound gel (Diagramma, Switzerland) was applied to the mouse head for ultrasonic coupling and the animals were wrapped in a polyethylene membrane. The mouse holder was 
placed in an imaging chamber filled with water and kept at $36.5^{\circ} \mathrm{C}$. Images were acquired at 10 wavelengths (i.e., 680 , $685,690,695,700,715,730,760,800$, and $850 \mathrm{~nm})$, for 35 to 40 consecutive slices with a step size of $0.3 \mathrm{~mm}$ and 10 averages, lasting $\sim 5 \mathrm{~min}$.

\subsection{Near-Infrared Fluorescence Imaging}

NIRF imaging was performed with the Maestro 500 multispectral imaging system (Cambridge Research \& Instruments Inc.), as previously described. ${ }^{28}$ The system was equipped with a band-pass filter ( 615 to $665 \mathrm{~nm}$ ) for excitation. The fluorescence was detected by a CCD camera fitted with a long-pass filter $(>700 \mathrm{~nm})$. Fluorescence emission images were acquired by automatically increasing the emission filter wavelengths at $10 \mathrm{~nm}$ steps. For in vivo NIRF imaging of MMP, four tMCAO and three sham-operated mice were anesthetized with $3 \%$ and maintained at $1.5 \%$ isoflurane in a 1:4 oxygen/ air mixture. For ex vivo NIRF imaging, brains were subsequently removed under deep anesthesia without prior perfusion. Coronal brain slices of 1-mm thickness were cut in a brain matrix using a razor blade. Sections were placed on object holders wrapped with black tape. Acquired image cubes were spectrally unmixed using Maestro software (Cambridge Research \& Instruments Inc.). Regions of interest (RoIs) were selected over each section on the ex vivo brain slices (Fig. 4). Fluorescence intensity (F.I.) ratios were calculated by dividing the RoI values of the left ischemic (ipsilateral) hemisphere by that of the right (contralateral) hemisphere.

\subsection{Magnetic Resonance Imaging}

Data were acquired on a $7 \mathrm{~T}$ Bruker Pharmascan (Bruker BioSpin $\mathrm{GmbH}$, Germany), equipped with a volume resonator operating in quadrature mode for excitation and a four element phased array surface coil for signal reception. During MRI acquisition, mice were kept under $1.5 \%$ isoflurane anesthesia in a $1: 4$ oxygen/air mixture. Body temperature was monitored with a rectal temperature probe (MLT415, ADInstruments) and kept at $36.5^{\circ} \mathrm{C} \pm 0.5^{\circ} \mathrm{C}$ using a warm water circuit integrated into the animal support (Bruker BioSpin GmbH, Germany). $\mathrm{T}_{2}$-weighted $\mathrm{MR}$ images were obtained using a spin echo sequence (TurboRARE) with echo time $3 \mathrm{~ms}$, repetition time $6 \mathrm{~ms}, 100$ averages, slice thickness $1 \mathrm{~mm}$, field-of-view $2.56 \times 1.28 \mathrm{~cm}^{2}$, matrix size $256 \times 128$, giving an in-plane resolution of $100 \mu \mathrm{m} \times 100 \mu \mathrm{m}$. For DWI, a four-shot spin echo-echo planar imaging sequence with echo time $=28 \mathrm{~ms}$, repetition time $=3000 \mathrm{~ms}$ was used. Twelve axial slices with a slice thickness of $1 \mathrm{~mm}$ and slice gap of $0.3 \mathrm{~mm}$ were acquired with a field-of-view of $3.3 \times 2 \mathrm{~cm}^{2}$ and a matrix size of $128 \times 128$, resulting in a nominal voxel size of $258 \mu \mathrm{m} \times$ $156 \mu \mathrm{m}$. Diffusion-encoding was applied in the $x$-, $y$-, and $z-$ directions with $b$-values of $100,200,400,600,800$, and $1000 \mathrm{~s} / \mathrm{mm}^{2}$, respectively, acquisition time $3 \mathrm{~min} 48 \mathrm{~s}$.

\subsection{Magnetic Resonance Image Reconstruction}

Maps of the apparent diffusion coefficient (ADC) were calculated on a pixel-by-pixel basis by linear regression analysis using the model function in Paravison 6 (Bruker Biospin $\mathrm{GmbH}$, Germany):

$$
\ln \left[S(b) / S_{0}\right]=-b \times \mathrm{ADC},
$$

where $S(b)$ is the pixel intensity as a function of $b$ and $S_{0}$ is the signal intensity of the pixel with zero diffusion gradient amplitude $(b=0)$. The ischemic lesion was determined as an area of significant reduction of the ADC value compared with the unaffected, contralateral side. On $\mathrm{T}_{2}$-weighted images, the lesion was determined as an area of hyperintensities compared with the contralateral side.

\subsection{MSOT Image Reconstruction and Spectral Unmixing}

MSOT images were reconstructed using a model-based algorithm, size $20 \mathrm{~mm}$, resolution $100 \mu \mathrm{m}$, and filter range from $50.0 \mathrm{kHz}$ to $7 \mathrm{MHz}$. The model-based reconstruction incorporates a detailed model of detection geometry that allows for more quantifiable images. For the multispectral processing, linear unmixing methods with a non-negative constraint imposed during inversion were applied to resolve signals from the MMP probe, deoxygenated $(\mathrm{Hb})$ and oxygenated hemoglobin $\left(\mathrm{HbO}_{2}\right)$, as described previously. ${ }^{27}$ The linear regression method uses known spectra for fitting and allows for reproducible results across studies. Tissue oxygen saturation $\left(\mathrm{SO}_{2}\right)$ was calculated by

$$
\mathrm{SO}_{2}=\frac{\mathrm{HbO}_{2}}{\mathrm{Hb}+\mathrm{HbO}_{2}} \times 100
$$

and

$\mathrm{SO}_{2}$ ipsi/contra $[\%]=\frac{\mathrm{SO}_{2} \text { ipsi }}{\mathrm{SO}_{2} \text { contralateral }} \times 100$.

\subsection{Coregistration of MSOT and MRI Images}

Functional MSOT images showing $\mathrm{Hb}, \mathrm{HbO}_{2}$, and MMP distribution share the same coordinates with the intrinsic anatomical images. This allows for registering the anatomical images obtained from MSOT to $\mathrm{T}_{2}$-weighted images/ADC maps and for applying the same transformation to all the functional images. Registration of MSOT and MR image was performed using a three-step strategy with MATLAB (R2015a, Mathworks). (The archived version of the code can be freely accessed and executed through Code Ocean: https://codeocean.com/ 2018/02/20/mri-msot-coregistration-matlab-code-demo.) First, the anatomical image was realigned with the $\mathrm{T}_{2}$-weighted MR image obtained at $48 \mathrm{~h}$ after reperfusion (or with the ADC maps at $1 \mathrm{~h}$ during occlusion) along the longitudinal axis. The alignment was performed under the assumption that, during measurement, the animal holder in MSOT moved along the $z$ axis, parallel to the central axis of the MRI scanner bore. Anatomical landmarks, such as the eye and brain structures, were selected for alignment. Linear interpolation was applied to MSOT images to generate the same number of frames as MRI for further processing. Second, nonreflective similarity transformation was applied to MSOT images after processing MR images. The transformation matrix was calculated based on the least square algorithm after manually selecting at least six pairs of landmarks in both MSOT and MR images. Rotation, scaling, and transformation were applied, with angles and shapes being preserved during registration. Finally, different MSOT maps ( $\mathrm{Hb}, \mathrm{HbO}_{2}$, and MMP) were registered with the corresponding MR image. The images were overlaid with multiple channels. RoIs were drawn over the lesion in the cortex, 


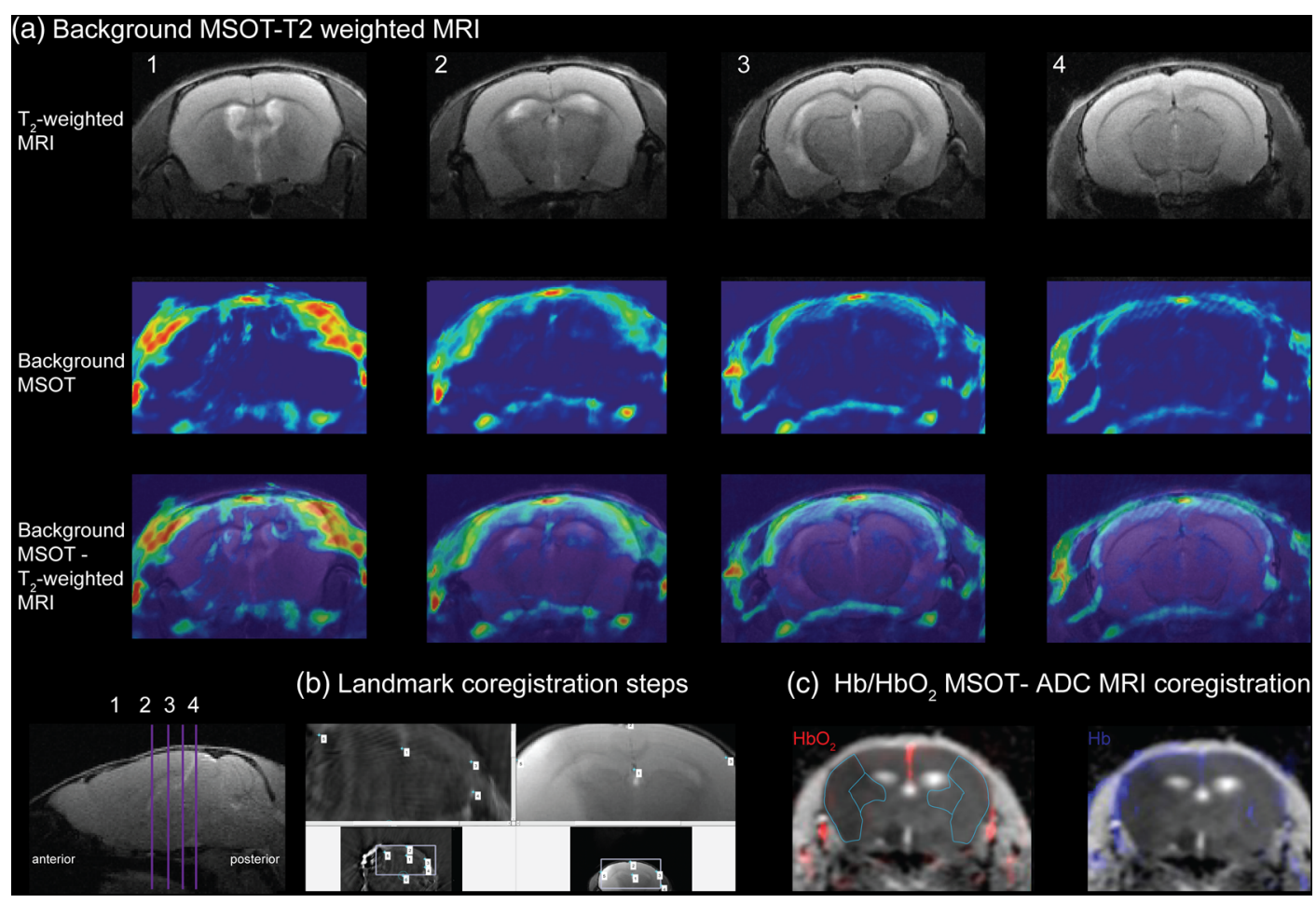

Fig. 1 Registration procedure: (a) coregistration of cross-sectional axial background MSOT and $\mathrm{T}_{2}$-weighted MR images; (b) landmark coregistration; and (c) resulting coregistered MSOT image and Rols, including ischemic lesion (whole, cortex, and striatum) in the ipsilateral and nonaffected contralateral hemisphere.

striatum, and the whole lesion in the ipsilateral and contralateral side on the axial overlaid MSOT/MR images using ImageJ $(\mathrm{NIH})$. The focal ischemic lesions were identified on the $\mathrm{T}_{2}$-weighted $\mathrm{MR}$ images and ADC maps. The size of RoIs was $\sim 3$ to $10 \mathrm{~mm}^{2}$ for the cortical-infarct and 5 to $10 \mathrm{~mm}^{2}$ for the striatal infarct and the corresponding contralateral areas (Fig. 1).

For assessing the depth dependence of MSOT signals, five parallel lines, each at two positions perpendicular to the cortex, were drawn on the $\mathrm{HbO}_{2}$ image in the contralateral hemisphere of one tMCAO mouse (illustrated in Fig. 5). The intensity (MSOT. a.u.) in relation to the distance (depth) from the cortex/surface of signal was analyzed.

\subsection{Triphenyltetrazolium Chloride Staining}

To assess the ischemic lesion severity in the tMCAO mice and in the sham-operated mice, staining with TTC staining was performed. Brain slices were incubated in a $2.5 \%$ TTC solution (Sigma-Aldrich) at $37^{\circ} \mathrm{C}$ for $3 \mathrm{~min}$. Pictures of stained samples were taken and analyzed by drawing RoIs over the contralateral side and the nonischemic ipsilateral side using ImageJ.

\subsection{Statistics}

Unpaired two-tail student's $t$ test with Welch's correction was used (Graphpad Prism 6.0, California) for comparing the difference between tMCAO and sham-operated mice. ANOVA with Turkey multiple comparison tested for the difference between sham-injected, tMCAO injected, and noninjected with MMP probe. Paired $t$ test was used for comparison of data between the ipsilateral and contralateral hemisphere. Unpaired two-tail student $t$ test with Welch's correction was used for comparison of
NIRF imaging data between tMCAO and sham-operated mice. All data are present as mean \pm standard deviation. Significance was set at $*, \# p<0.05$ and $* * *, \# \# \# p<0.001$.

\section{Results}

Data have been made available in a data repository (Ref. 29).

\subsection{In Vivo MSOT of Oxygenation during Occlusion and $48 \mathrm{H}$ After}

During occlusion, the lesions were visible on the ADC maps calculated from DWI, but not on the $\mathrm{T}_{2}$-weighted MR images, as expected. Thus, ADC maps were applied in the coregistration with images from MSOT for the hyperacute group. $T_{2}$-weighted MR images, which gave a better anatomical contrast for the lesions (discernable later than ADC maps), were used for coregistration for the group assessed at $48 \mathrm{~h}$ after reperfusion. The same transformations were subsequently applied to $\mathrm{Hb}, \mathrm{HbO}_{2}$, and MMP MSOT images. Figure 1 illustrates the MSOT-MRI coregistrated image that we obtained following a three-step strategy.

To assess the influence of acute tMCAO on brain regional tissue oxygenation, we measured the $\mathrm{HbO}_{2} / \mathrm{Hb}$ level in the brain of tMCAO and sham-operated mice during occlusion and at $48 \mathrm{~h}$ after reperfusion noninvasively in vivo using MSOT and calculated $\mathrm{SO}_{2}$ (Fig. 2). Lower $\mathrm{SO}_{2}$ ipsi/ $\mathrm{SO}_{2}$ contra ratios [\%] were detected in the lesion of tMCAO $(n=9)$ compared with sham-operated mice $(n=9) \quad(52.5 \pm 23.1 \%$, versus $98.6 \pm 18.3 \%, \quad p=0.0003)$ and in the cortical lesion $(44.9 \pm 28.5 \%$ versus $91.6 \pm 16.5 \%, p=0.0006)$, indicating severe focal ischemia. $\mathrm{SO}_{2}$ ipsi $/ \mathrm{SO}_{2}$ contra [\%] in the hemisphere and cortical brain region of the tMCAO animals were 

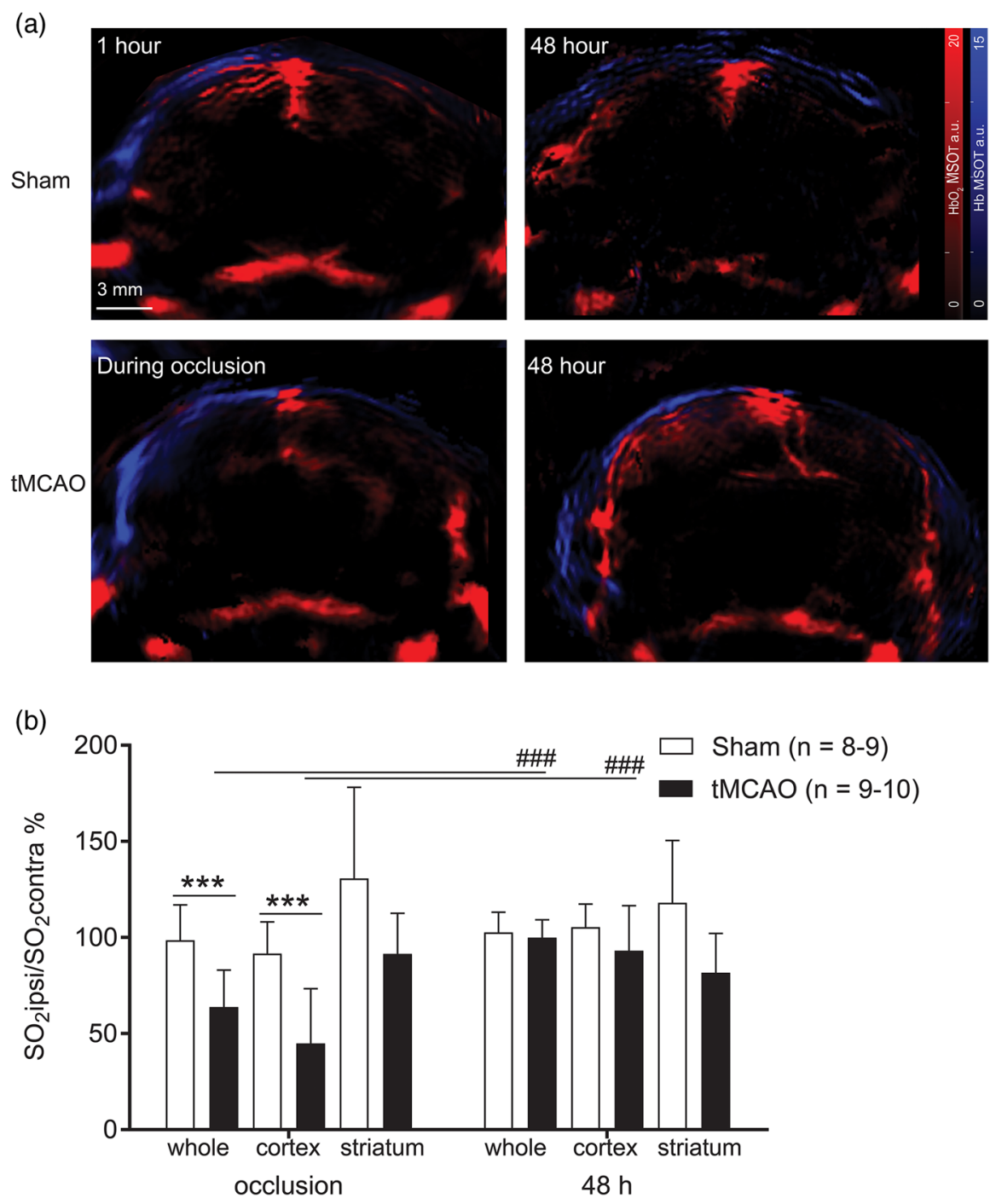

Fig. 2 In vivo assessment of brain oxygenation with MSOT in IMCAO and sham-operated mice. (a) $\mathrm{Hb}$ and $\mathrm{HbO}_{2}$ images of tMCAO and sham-operated mice during occlusion and at $48 \mathrm{~h}$ (coronal view, approximately Bregma $-0.58 \pm 0.3 \mathrm{~mm}$ ); unmixed signal from $\mathrm{HbO}_{2}$ (red) and $\mathrm{Hb}$ (blue); (b) reduced tissue $\mathrm{SO}_{2}$ in the whole lesion in the ipsilateral hemisphere and in the cortex compared with contralateral hemisphere in the brain of tMCAO compared with sham-operated mice during occlusion, with no differences between regions of groups at $48 \mathrm{~h}$; results are mean \pm standard deviation, ${ }^{\star \star \star} p<0.001$, tMCAO mice compared with sham-operated mice; \#\#\#p<0.001, tMCAO mice during occlusion compared with $48 \mathrm{~h}$; one way ANOVA with Turkey multiple comparison; tMCAO, Hb, deoxygenated hemoglobin; $\mathrm{HbO}_{2}$, oxygenated hemoglobin; $\mathrm{SO}_{2}$, oxygen saturation rate; MSOT, multispectral optoacoustic imaging;

significantly higher in the $48 \mathrm{~h}$ after reperfusion $(99.9 \pm 9.4 \%$, $n=9)$ compared with during occlusion $(93.1 \pm 23.5 \%$, $n=10$ ), and similar to the level of sham-operated mice at $48 \mathrm{~h}$ after reperfusion $(102.0 \pm 10.5 \%, n=8)$.

\subsection{In Vitro NIRF Imaging and MSOT of MMP Activated and Nonactivated Probe}

Analysis of NIRF images of the phantom indicated a threefold increase in the intensity of the MMP-activatable probe after activation with human recombinant MMP-9 compared with that of the nonactivated probe [F.I. average counts over the field, $232.2 \pm 31.8$ versus $77.5 \pm 10.2$ counts, $* * p=0.0086$; Fig. 3(a)]. Similarly, analysis of the MMP probe phantom measured with MSOT showed a three- to fourfold increase in the optoacoustic signal of the MMP activated probe [19196.0 \pm 3692.2 versus $5546.3 \pm 2625.6$ MSOT a.u., $p=0.0004$; Fig. 3(b)].

\subsection{In Vivo MSOT Imaging of MMP Activity in tMCAO Mice}

To reveal MMP activity in vivo, the MMP-activatable probe was injected into tMCAO and sham-operated mice as controls. tMCAO mice without the MMP activatable probe injection were measured as additional controls to assess the potential influence of tissue changes on MMP signal unrelated to the presence of the probe. Mice were assessed with MSOT $48 \mathrm{~h}$ after reperfusion with in-house generated MMP spectra and followed by MRI (Fig. 3). Significantly, higher unmixed MMP 
(a)

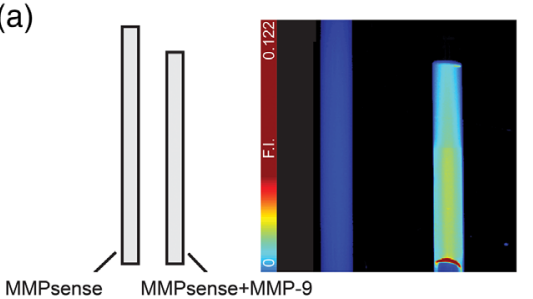

(c)

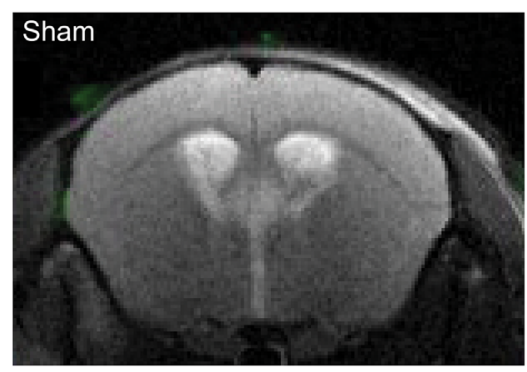

(b)
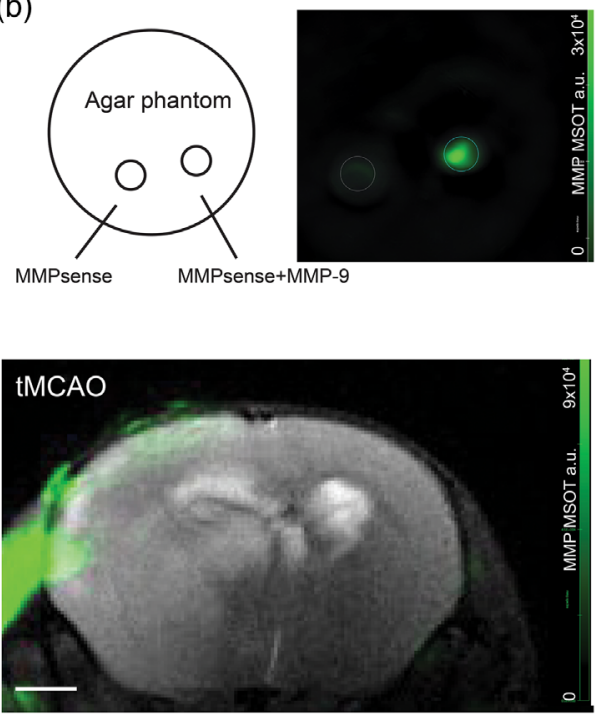

(d)

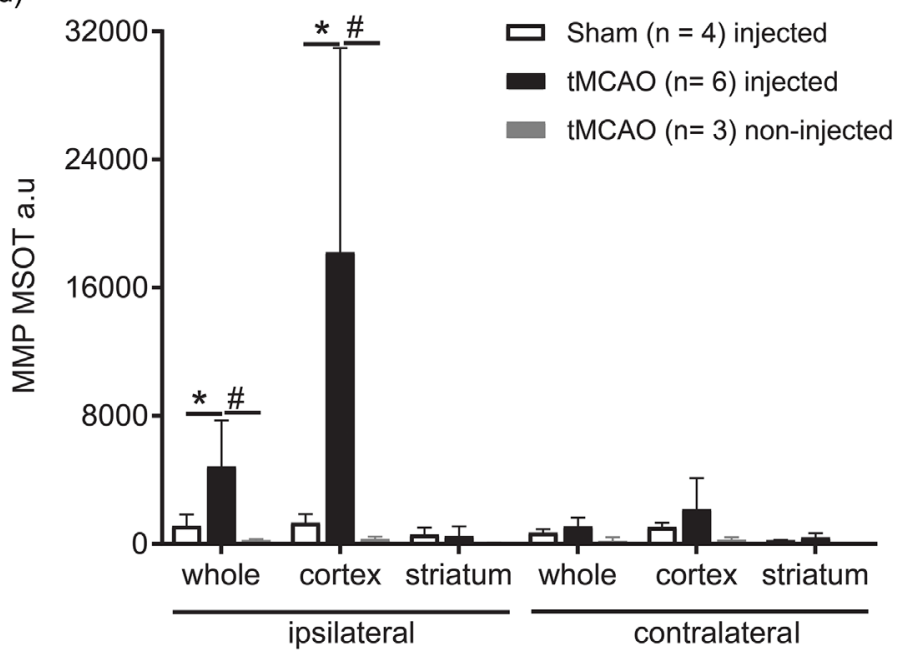

Fig. 3 In vivo MSOT assessment of MMP activity in phantom and brain of sham-operated and tMCAO mouse at $48 \mathrm{~h}$ after reperfusion. ( $\mathrm{a}$ and $\mathrm{b}$ ) NIRF imaging and MSOT of activated (human MMP-9) and unactivated MMP probe in a phantom, scale: F.I. 0 to 0.122 ; (c) sham and tMCAO mouse injected with MMP-activatable probe $48 \mathrm{~h}$ after reperfusion (approximately at Bregma $-0.58 \pm 0.3 \mathrm{~mm}$ ) with unmixed signal from MMP (green) overlaid on $T_{2}$-weighted MR image, scale 0 to $9 \times 10^{4}$ MSOT a.u.; (d) quantification of MSOT MMP signal in the ipsi/contralateral in the whole lesion, cortex, and striatum; results are Mean \pm standard deviation, ${ }^{*} p<0.05$, sham-injected compared with tMCAO-injected; $\# p<0.05$, to tMCAO-injected compared with tMCAO noninjected. One-way ANOVA with Turkey multiple comparison. F.I., fluorescence intensity; MMP, matrix metalloproteinase; tMCAO, transient middle cerebral artery occlusion.

intensities were observed in the ischemic ipsilateral hemisphere $(4738.7 \pm 2867.8$ MSOT a.u., $n=5)$ of tMCAO injected, compared with the nonischemic contralateral hemisphere $(1102.7 \pm 534.0$ MSOT a.u., $n=5, p=0.0309)$ and compared with sham-injected (1138.4 \pm 709.7 MSOT a.u., $n=4$, $p=0.0479)$. The MMP signals were higher in the cortical regions compared with that in the striatum and were lower than in all groups: tMCAO injected $(494.2 \pm 601.5, n=5)$ in the striatum similar to that in sham-operated injected mice $(584.9 \pm 442.2, n=4, p=0.9566)$. The noninjected tMCAO mice showed low level of signals in both the ipsilateral $(243.6 \pm 67.0, n=3)$ and contralateral hemisphere (201.1 \pm 226.5, $n=3$ ), significantly lower than that in the ipsilateral hemisphere of tMCAO injected (4837.7 $\pm 2867.8, p=0.0258)$.
Signals were also detected in extracerebral tissue of the mouse head in tMCAO and sham-operated mice injected with the MMP-activatable probe, likely due to MMP activation in muscles due to ligation of the external carotid artery. ${ }^{23}$

\subsection{In Vivo and Ex Vivo NIRF Imaging of MMP and TTC Staining in tMCAO Mouse Brains}

After MSOT, whole brains were removed from the skull for ex vivo NIRF imaging of the mouse brain and brain slices to assess the distribution of the activated MMP probe (Fig. 4). Higher MMP fluorescence intensities were detected in the ipsilateral lesioned hemisphere compared with contralateral hemisphere of tMCAO mice injected with the MMP-activatable 
(a)

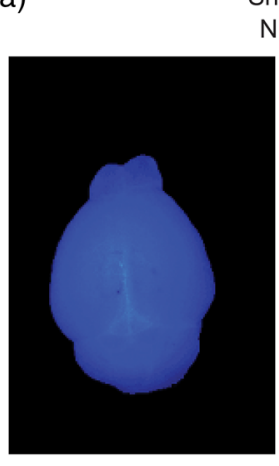

Sham

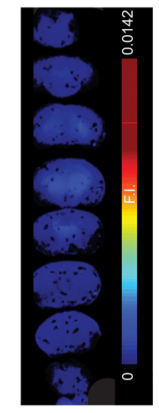

(b)

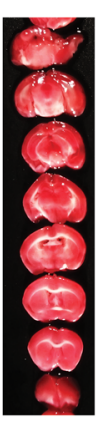

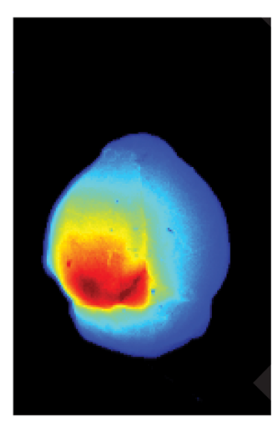

tMCAO NIRF

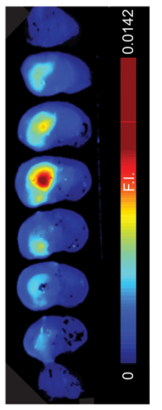

(c)

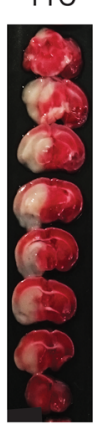

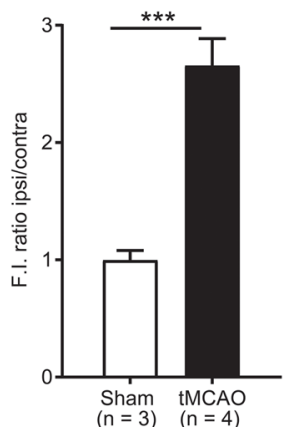

Fig. 4 Ex vivo NIRF imaging of MMP signal in the brain of sham-operated and tMCAO mice at $48 \mathrm{~h}$ after reperfusion. ( $a$ and $b)$ NIRF imaging of the brain, NIRF imaging, and TTC staining on the coronal brain slices, scale 0 to 0.0142 ; (c) quantification of MMP signals using ex vivo NIRF imaging in $\mathrm{tMCAO}(n=4)$ and sham $(n=3),{ }^{* \star *} p<0.001$, unpaired two tail student's $t$ test with Welch's correction. MMP, matrix metalloproteinase; tMCAO, transient middle cerebral artery occlusion; NIRF, near infrared fluorescence; F.I., fluorescence intensity;

(a)

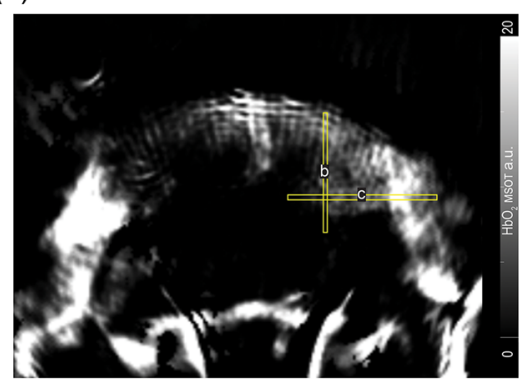

(b)

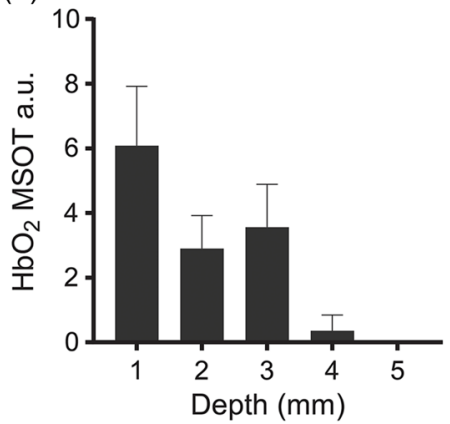

(c)

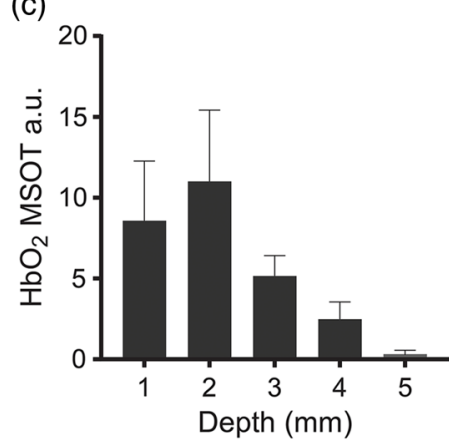

Fig. 5 Depth analysis of MSOT signal in a representative tMCAO mouse brain. (a) Coronal view of MSOT image of $\mathrm{HbO}_{2}$ in a tMCAO mouse brain at $48 \mathrm{~h}$ after reperfusion and illustration of Rol used for depth analysis, scale 0 to 20 MSOT a.u. (b and c) Plotting of mean signal intensity in relation to depth $(\mathrm{mm})$ in two Rols as illustrated in (a). $\mathrm{HbO}_{2}$, oxygenated hemoglobin; tMCAO, transient middle cerebral artery occlusion; MSOT, multispectral optoacoustic imaging;

probe (ipsi/contralateral ratio $2.7 \pm 0.2$ ). No difference was observed between the fluorescence intensity in the two hemispheres of sham-operated mice injected with the MMP-activatable probe (ipsi/contralateral ratio $1.0 \pm 0.01, p<0.0001$ compared with tMCAO). Ex vivo NIRF imaging of brain sections of tMCAO mice validated the high fluorescence intensities to areas of nonviable tissue as demarcated by TTC staining.

\subsubsection{Depth dependence of MSOT signal}

To analyze the depth dependence of MSOT, signal intensity was plotted as a function of depth at two different locations in the right contralateral hemisphere in the brain of a tMCAO mouse. The $\mathrm{HbO}_{2}$ signals were detectable across the cortical layers up to $\sim 3$ to $4 \mathrm{~mm}$ at two positions and decreased thereafter with increasing depth (Fig. 5).

\section{Discussion}

The pathophysiology of ischemic stroke is complex, involving a myriad of cellular and molecular pathways, where hemodynamic alterations and inflammation play a prominent role in ischemic tissue damage. ${ }^{2}$ We demonstrated a reduced tissue oxygenation during tMCAO and restoration of normal tissue oxygenation at $48 \mathrm{~h}$ after reperfusion with MSOT in vivo. We showed that the in vivo MSOT detection of the MMP signal predominantly located within the ischemic territory of tMCAO mouse brain, corroborated by ex vivo NIRF imaging of brain tissue slices.

Because MSOT has inherently low soft tissue contrast, we incorporated structural information MRI measurements to inform RoI analysis of MSOT data (ADC maps for the hyperacute stage and $\mathrm{T}_{2}$-weighted MRI for the subacute stage). Diffusional changes on ADC maps are caused by shifts of water from the extracellular to intracellular compartment due to the developing cytotoxic edema ${ }^{30}$ while increases of regional $T_{2}$ reflect formation of vasogenic edema and liquefactive necrosis. ${ }^{31}$ In addition, MRI is very versatile and can deliver a variety of other measures of tissue integrity (e.g., fluid-attenuated inversion recovery, magnetization transfer, and diffusion tensor imaging for white matter integrity, susceptibilityweighted imaging for hemorrhagic transformation) and hemodynamics (e.g., arterial spin labeling for CBF, cerebral blood volume, vascular reserve capacity), which cannot be attained by optoacoustic imaging in the same way and that can be very useful in experimental stroke research. MRI may also serves as structural prior information for a better reconstruction algorithm 
for MSOT image. ${ }^{32}$ We applied semiautomatic registration based on nonreflective similarity transformation with multiple point pairs to reduce manual localization errors of the landmarks. A similar framework has been used in registration of MSOT with MR images of a glioblastoma mouse model. ${ }^{33}$ As the accuracy of the registration algorithm largely depends on landmark selection, more precise registration would be achieved using artificial fiducials, such as agar-based globules, visible in both MSOT and MRI. Alternatively, intensity-based methods to maximize the similarity measure between images and the mutual-information method could address the issues in multimodal imaging registration. ${ }^{34,35}$ There is a lack of overlap between the $\mathrm{Hb}$ and $\mathrm{HbO}_{2}$ signal due to physiological and display reasons: in arteries, the oxygen saturation is almost $100 \%$ and, thus, the $\mathrm{HbO}_{2}$ signal should dominate. In the veins and capillaries (that are contained in tissue voxels), the oxygen saturation is $85 \%$ to $90 \%$, which should give a mixture of $\mathrm{Hb}$ and $\mathrm{HbO}_{2}$ signals, where $\mathrm{Hb}$ accounts for $10 \%$ to $15 \%$ and $\mathrm{HbO}_{2}$ for $85 \%$ to $90 \%$. $^{36-40}$ Therefore, in general, there is less intense $\mathrm{Hb}$ signal compared with $\mathrm{HbO}_{2}$. Moreover, blue color is usually perceived as less intense than red color. MSOT signal observed outside the brain is mainly due to the presence of extracerebral vessels and tissue in the mouse head. Major contributors are the external carotid arteries, the petrosquamosal sinus, facial vein as blood vessels, and the temporal and masseter muscles surrounding the skull. In addition, MSOT signal outside the brain region is due to surface-weighted artifacts caused by the reconstruction procedure and not coregistration. A similar phenomenon was observed in a previous MSOT study using similar registration workflow. ${ }^{33}$

Other optical imaging methods for brain hemodynamics measurement, such as optoacoustic microscopy ${ }^{18,36,41,42}$ and single-impulse panoramic optoacoustic computed tomography, ${ }^{43,44}$ have been applied. We used MSOT to assess regional tissue oxygenation in the brain during hyperacute and acute stages of ischemia. It should be noted that while MSOT can provide metrics of oxygen saturation based on measurements of $\mathrm{Hb}$ and $\mathrm{HbO}_{2}$, this method does not provide the absolute tissue oxygenation value, which requires knowledge of the light fluence distribution, system response, and Grüneisen parameter. ${ }^{45}$ Moreover, light propagating in deep tissue attains a spectrum that varies with location due to wavelength-dependent fluence attenuation, which can impact the accuracy of oxygen quantification. An improved algorithm to account for these effects has been described but has not become widely available. ${ }^{46}$ We showed a reduction of tissue $\mathrm{SO}_{2}$ in the whole ischemic lesion and the cortex during hyperacute ischemia by $\sim 50 \%$. Oxygen extraction is elevated to maintain normal neuronal metabolism when $\mathrm{CBF}$ is reduced. Residual oxygenation may result from collateral blood supply. ${ }^{47}$ The level of tissue $\mathrm{SO}_{2}$ in tMCAO returned to normal subacutely. This may indicate normalization of tissue oxygen extraction when blood flow to the middle cerebral artery territory is restored. The exact time point when tissue oxygenation starts to normalize remains to be investigated. While penumbral neurons are functionally compromised, they are salvageable from cell death if oxygen supply is restored. Hence, therapeutic options rely mainly on recanalization strategies by pharmacological and/or mechanical thrombolysis.

Inflammatory processes occur secondary to ischemia and reperfusion and are major contributors to tissue injury but also to repair and regeneration. Transient ischemia and reperfusion induce pathological upregulation and activity of MMPs. ${ }^{48,49}$ The phantom experiment with probe activated in vitro showed that the activated MMP probe can be sensitively detected both with NIRF imaging and MSOT. The ability to detect MMP activated fluorescent sensors in a postmortem tissue with MSOT has been reported previously. ${ }^{23,24,50}$ The validations of the MMP activatable probe for use in the tMCAO model, i.e., in vivo NIRF imaging in MMP knockout mice, and comparison of MMP inhibitor and nonactivatable probe, have been investigated thoroughly in our previous study. ${ }^{23}$ Compared with planar NIRF imaging studies for detecting MMP, ${ }^{28,51-53}$ the tomographic reconstruction of MSOT allows discriminating the contribution from different tissue compartments, which is not feasible in planar NIRF imaging. And while NIRF imaging and MSOT both work with light in the NIR range, compared with NIRF imaging, MSOT is not sensitive to light scattering and can thus achieve much higher spatial resolution. This has, for example, allowed the discrimination of the MMP signal from cerebral and extracerebral tissue, which is induced in the temporal muscle by the surgical procedures. ${ }^{23}$ However, it is noted that the distribution of signal intensity in the brain differs between in vivo MSOT imaging and ex vivo NIRF imaging of sections: the highest NIRF intensities locate in the ischemic striatum with NIRF imaging, while the highest intensities in the ischemic cortex were detected with MSOT. This difference may be explained by the signal formation mechanism. In ex vivo NIRF imaging of brain tissue, the surrounding tissues are removed, and deep brain regions like the striatum are similarly illuminated as areas near the surface like the cortex. By contrast, less excitation light reaches deep brain regions in in vivo MSOT due to absorption of extracerebral tissue like the skin, muscle, and scalp and within the brain, as supported by the depth dependency analysis of the MSOT measure of $\mathrm{HbO}_{2}$ signal in mouse brain (Fig. 5).

There are several potential sources of background signals in MSOT measurement of MMP in vivo in mouse, including endogenous change in ischemic tissue, MSOT system baseline noise, and background by nonactivated and activated probe in the circulation. In the tMCAO mice, the blood-brain barrier might be impaired, also as a consequence of MMP activity, leading to an accumulation of the activated probe. Further studies would need to elucidate the contribution from blood-brain barrier leakage on the generated contrast.

In conclusion, MRI provides information on structural changes with high soft tissue contrast for demarcating the ischemic lesion. MSOT provides limited anatomical information but enables characterization of physiological and molecular changes in and around the ischemic lesion with high sensitivity and resolution. The combination of the two techniques provides complementary in vivo information on important pathophysiology of the mouse model noninvasively. The techniques can thus be useful for multiplexing studies of the disease mechanism in the same animal and to test the effects of potential treatments.

\section{Disclosures}

The authors declare no financial or personal conflicts of interest.

\section{Acknowledgments}

The author would like to thank Diana Rita Kindler and Jael Xandy at the Institute for Biomedical Engineering, ETH Zurich/Univerity of Zurich, and Dr. Thomas Sardella at iThera Medical for technical assistance. This work was funded 
by the University of Zurich and the ETH Zurich Foundation through a Seed Grant of "University Medicine Zurich/ Hochschulmedizin Zürich" and by funding from the Olga Mayenfisch Stiftung.

\section{References}

1. J. Astrup, "Energy-requiring cell functions in the ischemic brain. Their critical supply and possible inhibition in protective therapy," J. Neurosurg. 56(4), 482-497 (1982).

2. U. Dirnagl, C. Iadecola, and M. A. Moskowitz, "Pathobiology of ischaemic stroke: an integrated view," Trends Neurosci. 22(9), 391-397 (1999).

3. P. Y. Cheung et al., "Matrix metalloproteinase-2 contributes to ischemiareperfusion injury in the heart," Circulation 101(15), 1833-1839 (2000).

4. K. P. Park et al., "Plasma and brain matrix metalloproteinase-9 after acute focal cerebral ischemia in rats," Stroke 40(8), 2836-2842 (2009).

5. G. A. Rosenberg, E. Y. Estrada, and J. E. Dencoff, "Matrix metalloproteinases and TIMPs are associated with blood-brain barrier opening after reperfusion in rat brain," Stroke 29(10), 2189-2195 (1998).

6. J. H. Heo et al., "Matrix metalloproteinases increase very early during experimental focal cerebral ischemia," J. Cereb. Blood Flow Metab. 19(6), 624-633 (1999).

7. M. Asahi et al., "Effects of matrix metalloproteinase- 9 gene knock-out on the proteolysis of blood-brain barrier and white matter components after cerebral ischemia," J. Neurosci. 21(19), 7724-7732 (2001).

8. V. W. Yong, "Metalloproteinases: mediators of pathology and regeneration in the CNS," Nat. Rev. Neurosci. 6(12), 931-944 (2005).

9. B. Q. Zhao et al., "Role of matrix metalloproteinases in delayed cortical responses after stroke," Nat. Med. 12(4), 441-445 (2006).

10. G. C. Jickling et al., "Hemorrhagic transformation after ischemic stroke in animals and humans," J. Cereb. Blood Flow Metab. 34(2), 185-199 (2014).

11. B. Chelluboina et al., "Matrix metalloproteinase-12 induces blood-brain barrier damage after focal cerebral ischemia," Stroke 46(12), 3523-3531 (2015).

12. Z. Gu et al., "A highly specific inhibitor of matrix metalloproteinase-9 rescues laminin from proteolysis and neurons from apoptosis in transient focal cerebral ischemia," J. Neurosci. 25(27), 6401-6408 (2005).

13. R. A. Kruger et al., "Photoacoustic ultrasound (PAUS)-reconstruction tomography," Med. Phys. 22(10), 1605-1609 (1995).

14. D. Razansky, C. Vinegoni, and V. Ntziachristos, "Multispectral photoacoustic imaging of fluorochromes in small animals," Opt. Lett. 32(19), 2891-2893 (2007).

15. X. Wang et al., "Noninvasive laser-induced photoacoustic tomography for structural and functional in vivo imaging of the brain," Nat. Biotechnol. 21(7), 803-806 (2003).

16. S. Gottschalk et al., "Noninvasive real-time visualization of multiple cerebral hemodynamic parameters in whole mouse brains using fivedimensional optoacoustic tomography," J. Cereb. Blood Flow Metab. 35(4), 531-535 (2015).

17. L. V. Wang and S. Hu, "Photoacoustic tomography: in vivo imaging from organelles to organs," Science 335(6075), 1458-1462 (2012).

18. J. Yao et al., "Noninvasive photoacoustic computed tomography of mouse brain metabolism in vivo," Neuroimage 64, 257-266 (2013).

19. S. Gottschalk et al., "Correlation between volumetric oxygenation responses and electrophysiology identifies deep thalamocortical activity during epileptic seizures," Neurophotonics 4(1), 011007 (2017).

20. M. Kneipp et al., "Functional real-time optoacoustic imaging of middle cerebral artery occlusion in mice," PLoS One 9(4), e96118 (2014).

21. J. Weber, P. C. Beard, and S. E. Bohndiek, "Contrast agents for molecular photoacoustic imaging," Nat. Methods 13(8), 639-650 (2016).

22. G. P. Luke, D. Yeager, and S. Y. Emelianov, "Biomedical applications of photoacoustic imaging with exogenous contrast agents," Ann. Biomed. Eng. 40(2), 422-437 (2012).

23. D. Razansky et al., "Multispectral optoacoustic tomography of matrix metalloproteinase activity in vulnerable human carotid plaques," Mol. Imaging Biol. 14(3), 277-285 (2012).

24. H. Qin et al., "Inflammation-targeted gold nanorods for intravascular photoacoustic imaging detection of matrix metalloproteinase-2
(MMP2) in atherosclerotic plaques," Nanomed.: Nanotechnol. Biol. Med. 12(7), 1765-1774 (2016).

25. V. Cortez-Retamozo et al., "Real-time assessment of inflammation and treatment response in a mouse model of allergic airway inflammation," J. Clin. Invest. 118(12), 4058-4066 (2008).

26. M. Vaas et al., "Extracerebral tissue damage in the intraluminal filament mouse model of middle cerebral artery occlusion," Front. Neurol. 8, 85 (2017).

27. S. Tzoumas et al., "Unmixing molecular agents from absorbing tissue in multispectral optoacoustic tomography," IEEE Trans. Med. Imaging 33(1), 48-60 (2014).

28. J. Klohs et al., "In vivo near-infrared fluorescence imaging of matrix metalloproteinase activity after cerebral ischemia," J. Cereb. Blood Flow Metab. 29(7), 1284-1292 (2009).

29. R. Ni et al., "MMP in tMCAO mouse brain," http://doi.org/10.6084/m9 .figshare.5501332.

30. H. Benveniste, L. W. Hedlund, and G. A. Johnson, "Mechanism of detection of acute cerebral ischemia in rats by diffusion-weighted magnetic resonance microscopy," Stroke 23(5), 746-754 (1992).

31. H. Kato et al., "Characterization of experimental ischemic brain edema utilizing proton nuclear magnetic resonance imaging," J. Cereb. Blood Flow Metab. 6(2), 212-221 (1986).

32. M. Niedre and V. Ntziachristos, "Elucidating structure and function in vivo with hybrid fluorescence and magnetic resonance imaging," Proc. IEEE 96(3), 382-396 (2008).

33. A. B. Attia et al., "Multispectral optoacoustic and MRI coregistration for molecular imaging of orthotopic model of human glioblastoma," J. Biophotonics 9(7), 701-708 (2016).

34. D. Rueckert et al., "Nonrigid registration using free-form deformations: application to breast MR images," IEEE Trans. Med. Imaging 18(8), 712-721 (1999).

35. J. P. Pluim, J. B. Maintz, and M. A. Viergever, "Mutual-informationbased registration of medical images: a survey," IEEE Trans. Med. Imaging 22(8), 986-1004 (2003).

36. R. Cao et al., "Functional and oxygen-metabolic photoacoustic microscopy of the awake mouse brain," Neuroimage 150, 77-87 (2017).

37. H. F. Zhang et al., "Imaging of hemoglobin oxygen saturation variations in single vessels in vivo using photoacoustic microscopy," Appl. Phys. Lett. 90(5), 053901 (2007).

38. X. Wang et al., "Noninvasive imaging of hemoglobin concentration and oxygenation in the rat brain using high-resolution photoacoustic tomography," J. Biomed. Opt. 11(2), 024015 (2006).

39. R. D. Shonat et al., "Near-simultaneous hemoglobin saturation and oxygen tension maps in mouse brain using an AOTF microscope," Biophys. J. 73(3), 1223-1231 (1997).

40. H. Kobayashi and N. Takizawa, "Oxygen saturation and $\mathrm{pH}$ changes in cremaster microvessels of the rat," Am. J. Physiol. 270(4 Pt 2), H1453H1461 (1996).

41. J. Yao et al., "High-speed label-free functional photoacoustic microscopy of mouse brain in action," Nat. Methods 12(5), 407-410 (2015).

42. L. Wang, K. Maslov, and L. V. Wang, "Single-cell label-free photoacoustic flowoxigraphy in vivo," Proc. Natl. Acad. Sci. U. S. A. 110(15), 5759-5764 (2013).

43. L. Li et al., "Single-impulse panoramic photoacoustic computed tomography of small-animal whole-body dynamics at high spatiotemporal resolution," Nat. Biomed. Eng. 1, 0071 (2017).

44. L. V. Wang, "Multiscale photoacoustic microscopy and computed tomography," Nat. Photonics 3(9), 503-509 (2009).

45. M. R. Tomaszewski et al., "Oxygen enhanced optoacoustic tomography (OE-OT) reveals vascular dynamics in murine models of prostate cancer," Theranostics 7(11), 2900-2913 (2017).

46. S. Tzoumas et al., "Eigenspectra optoacoustic tomography achieves quantitative blood oxygenation imaging deep in tissues," Nat. Commun. 7, 12121 (2016).

47. K. A. Hossmann, "The two pathophysiologies of focal brain ischemia: implications for translational stroke research," J. Cereb. Blood Flow Metab. 32(7), 1310-1316 (2012).

48. V. W. Yong et al., "Metalloproteinases in biology and pathology of the nervous system," Nat. Rev. Neurosci. 2(7), 502-511 (2001).

49. B. Zinnhardt et al., "Multimodal imaging reveals temporal and spatial microglia and matrix metalloproteinase activity after experimental stroke," J. Cereb. Blood Flow Metab. 35(11), 1711-1721 (2015). 
50. J. Levi et al., "Design, synthesis, and imaging of an activatable photoacoustic probe," J. Am. Chem. Soc. 132(32), 11264-11269 (2010).

51. M. Takamiya et al., "Strong neuroprotection with a novel platinum nanoparticle against ischemic stroke- and tissue plasminogen activator-related brain damages in mice," Neuroscience 221, 47-55 (2012).

52. P. A. Barber et al., "Infrared optical imaging of matrix metalloproteinases (MMPs) up regulation following ischemia reperfusion is ameliorated by hypothermia," BMC Neurosci. 13, 76 (2012).

53. F. Tian et al., "In vivo optical imaging correlates with improvement of cerebral ischemia treated by intravenous bone marrow stromal cells (BMSCs) and edaravone," Neurol. Res. 35(10), 1051-1058 (2013).

Ruiqing $\mathrm{Ni}$ received her BSc degree in pharmacy at the Fudan University, Shanghai, China. She completed her PhD in medicine (Neuroscience programme) at the Karolinska Institutet, Stockholm, Sweden, and short-term JSPS fellowship postdoctral at the National Institute of Radiological Science Japan. Currently, she is a postdoctoral research at ETH Zurich/University of Zurich. Her research focuses on the development of positron emission tomography, optical imaging and magnetic resonance imaging biomarkers for early diagnosis of Alzheimer's disease.
Markus Vaas studied biology and received a $\mathrm{PhD}$ in biomedical engineering from the ETH Zürich, Switzerland. He is now a postdoctoral researcher at the University of Zurich. In his research he applies optical imaging techniques to study the cellular inflammatory response (in particular the role of neutrophils) after ischemic stroke.

Wuwei Ren received his BSc degree in biomedical engineering, Zhejiang University, China, and his MSc degree in medical imaging in the joint program of the Royal Institute of Technology and Karolinska Institute, Sweden. Currently, he is pursuing his PhD on the topic of image reconstruction of fluorescence tomography using MRI prior information.

Jan Klohs received his BSc degree in chemistry at the University of Wales, Swansea, United Kingdom. He completed his MSc and PhD degrees in medical neurosciences at the Charite -University Medicine Berlin, Germany. Currently, he is an assistant professor for preclinical imaging at the University of Zurich. His research focuses on the development of optical imaging and magnetic resonance imaging techniques, and investigations of preclinical models of brain disease. 